\title{
Effect of manipulation by maitland method in the treatment of adhesive capsulitis of the shoulder: case studies
}

\author{
Mafra Raiele Torres Oliveira1', Vivianne Camila Souza Bastos', Lívia Shirahige Gomes do Nascimento², \\ Maria de Fátima Alcântara Barros³, Antonio Geraldo Cidrão de Carvalho³, Miburge Bolivar Gois Junior, \\ Marcelo Renato Guerino ${ }^{5}$, Shirley Lima Campos ${ }^{5}$, Maria das Graças Rodrigues de Araújo $0^{5,6}$
}

\begin{abstract}
Introduction: Adhesive capsulitis, also called frozen shoulder, affects about 3-5\% of the population, especially in the age group 40 to 60 years, affecting more women and having an insidious onset. Objective: Analyze the isolated effect of joint manipulation by Maitland method in treating patients with adhesive capsulitis of the shoulder, the level of pain, range of motions of the shoulder and functional capacity. Method: It is about a cases studies in which four volunteers underwent protocol Maitland to shoulder for 15 sessions. The evaluation instruments used were the Visual Analog Scale, Goniometer and Shoulder Pain and Disability Index to measure the level of disability of the shoulder. Results: There was decreased pain from 55.5 to $80.0 \%$, increased all range of motions, especially flexion of the shoulder, with more than $100 \%$ gains in the four cases investigated and improved functional capacity between $27.9-91.9 \%$. Conclusion: These results suggest that the therapy had improved outcomes assessed, as clinically relevant contribution, but indicate the need to develop more studies to prove the effectiveness of the method isolated.
\end{abstract}

Key words: Adhesive capsulitis, Shoulder, Joint manipulation, Physiotherapy, Maitland.

\section{INTRODUCTION}

In Brazil, one study found the incidence of shoulder pain syndromes in $32.5 \%$ of patients aged $40-50$ years ${ }^{(1)}$. In this context, it highlights the Adhesive Capsulitis (AC), also called frozen shoulder ${ }^{(2)}$, which affects about $3-5 \%$ of the population geral ${ }^{(3)}$ mainly in the age group of $40-60$ years and affects more women having early insidious. ${ }^{(4)}$

There are controversies surrounding the diagnosis of AC, mainly due to uncertainty in its pathogenesis ${ }^{(5)}$, which can be primary and emerge in a few weeks without apparent cause or result from trauma ${ }^{(6)}$. Occur inflammatory and fibrous changes in the joint capsule and synovial lining causing retraction of the capsule and promoting joint stiffness. ${ }^{(3)}$

Thus, patients have as main feature the total loss of range of motion ( ROM) active and passive shoulder (7) mainly in flexion, abduction, external rotation and internal. ${ }^{(8)}$

The condition is also characterized by pain, even at rest, present in $40-50 \%$ of affected ${ }^{(9)}$, which may decrease the intensity of a few weeks or months, or progress until the shoulder immobility. ${ }^{(6)}$

The pain is associated with decreased functional capacity interferes with quality of life of affected patients. ${ }^{(10)}$
The condition involves three stages: the painful stage of freezing and thawing. ${ }^{(7)}$

There are several clinical, surgical, and physical therapy procedures carried out on these patients in order to relieve the pain and the functional movements to return. ${ }^{(10)}$

The physical therapy has a number of features, including therapeutic exercises, electrotherapy, massage therapy, pilates, osteopathy and global postural re-education. The joint mobilization accessories movements has physiological effect (analgesia proprioceptive feedback cartilage nutrition, etc.) that may be beneficial in these dysfunctions. ${ }^{(11)}$

The approach of Maitland method is based on a graded assessment and treatment system through passive oscillatory movements, rhythmic, graded in five levels that vary with the amplitude of the accessory movements normally present in the joints. ${ }^{(12)}$ Symptoms, movements and joint positions are tested in active movement, being considered any change in amplitude, rhythm, reproduction and pain arc. ${ }^{(13)}$

Although developed by Australian Geoffrey Maitland in $1970^{(14)}$, the isolated application of Maitland method to AC treatment still shows little evidence. In a recent systematic

\footnotetext{
Author Corresponding: Maria das Graças Rodrigues de Araújo. Department of Physiotherapy, Health Sciences Center, Federal University of Pernambuco- UFPE. Jornalista Aníbal Fernandes Avenue, S/N, Cidade Universitária; Recife - PE; Brasil; Zip Code: 58051-900. Phone: +55 (81) 21268939. E-mail: mgrodriguesaraujo@hotmail.com

${ }^{5}$ Department of Physiotherapy, UFPE - Universidade Federal de Pernambuco, Recife (PE), Brazil.

'Laboratory of Kinesiotherapy and Manual Therapeutic Resources, Program in Physical Therapy, UFPE - Universidade Federal de Pernambuco, Recife (PE), Brazil. Full list of author information is available at the end of the article.
}

Financial support: This research received no funding

Submission date 5 April 2016; Acceptance date 4 August 2016; Publication online date 22 August 2016 
review ${ }^{(6)}$ on the treatment of CA just one item included using the Maitland method and yet not alone, and in combination with other therapeutic exercises.

Randomized controlled trials using the same methodology ${ }^{(15.5)}$ which complicates the analysis of the effectiveness of the technique itself.

Therefore, the objective of this study is to analyze the effects of isolated application of joint mobilization by the Maitland method for treating patients with shoulder CA on the level of pain, ADM and functional capacity.

\section{METHOD}

It is a case study, developed in the Laboratory of Kinesiotherapy and Therapeutic Resource Manuals of Department of Physical Therapy, Universidade Federal de Pernambuco (DEFISIO - UFPE) in the period April to December 2013.

The volunteers were captured by disclosure through leaflet, written and television news. Twelve individuals interested in participating in the project sought the laboratory and underwent screening and compliance with the eligibility criteria.

The inclusion criteria were used: age between $40-60$ years, confirmed medical diagnosis of adhesive capsulitis of the shoulder and the absence of prior physical therapy. The exclusion criteria were: presence of cognitive or neurological disorders, presence of other injuries and orthopedic disorders associated with shoulder that make it impossible to performing surgery.

In this process, seven volunteers had confirmed diagnosis of $C A$, but 3 were excluded due to other injuries associated with shoulder two exclusions occurred by the presence of associated injuries to the superior glenoid labrum (SLAP); and a partial tear of the tendon of the supraspinatus muscle.

So four individuals were informed about the nature of the study and its stages, as well as the voluntary and the possibility of withdrawal during the research. In the end, all signed the the terms of consent free and Cleared up.

This study was approved by the Ethics Committee on Health Sciences Research Center of UFPE under CAAE Protocol: 03130172000-09.

\section{PROCEDURES}

The survey of demographic data was conducted through descriptive variables (gender, age, profession, the current disease history, affected limb, impairment of time, comorbidities and medications for pain relief and independent variables (pain, ADM and capacity functional) study .

The Visual Analogue Scale (VAS) is a simple tool that consists of a graduated scale from zero to ten and widely used to assess pain in both clinical situations as in research. ${ }^{(16)}$ In this study, data were collected at the beginning and end of each session, and the patient measure your pain, considering the scores 0-2 presence of mild pain , 3-7 moderate pain and severe pain 8-10.

The Visual Analogue Scale (VAS) is a simple tool that consists of a graduated scale from zero to ten and widely used to assess pain in both clinical settings and in research. ${ }^{(16)}$ In this study, data were collected at the beginning and end of each session, and the patient measure your pain, considering the scores 0-2 presence of mild pain, 3-7 moderate pain and severe pain 8-10.

The range of motion (ROM) was verified by goniometer (goniometer for large joints with protractor system from 0 을 to 360 ㅇ, $\mathrm{Carci}^{\circledR}$, São Paulo, Brazil) according to the recommendations of the Clinical Practice Guideline for Shoulder Pain and Mobility Deficits: Adhesive Capsulitis. ${ }^{(7)}$ Were collected three measures for flexion, abduction, external rotation and internal with final result consisted of an average.

Functional capacity was assessed using the Pain Disability Index and Shoulder (Shoulder Pain and Disability Index in the version for the Portuguese language in Brazil, SPADI, test-retest reliability: 0.90 to 0.94 ; internal consistency: $0.87-0.89$ ). Consists of 13 items distributed in the field of pain and function, each item scored 0-10 points, the final score of the questionnaire, and the score separately for each domain is converted into a percentage ranging from 0 to 100 , with the highest score indicating dysfunction worse condition. ${ }^{(17)}$

The protocol consisted of daily application of the Maitland method interval on weekends, totaling 15 sessions. The sessions were applied by a single researcher, previously trained, being composed of three repetitions for each movement lasting one minute of manipulation with one minute of rest ${ }^{(18)}$ using both high manipulations as low grade.

Each volunteer was positioned following: supine (DD), prone (DV) and after sitting three series for each movement.

\section{In supine:}

(1) The therapist supported the affected arm holding it with one hand and the other fixing the shoulder in the coracoid process to perform movements of the humerus toward the anterior capsule.

(2) It withdrew support in the coracoid process in order to perform movements of the humerus toward the lateral capsule.

\section{In prone:}

(1) Performed up the humeral movement toward the posterior capsule resting one hand on the scapula to stabilize the shoulder and the other on the affected arm.

(2) Performed to the humerus move toward posterior capsule resting one hand on the scapula to stabilize the shoulder and the other on the affected arm. 
In sitting position:

(1) With the support acromion - made up of the humerus move towards the top shell.

(2) With axillary support - made up of the humerus move toward lower capsule.

At the end of the sequence of the above movements, voluntary returned to $\mathrm{DD}$, the longitudinal accessory movements taking place, postero anterior, antero posterior and lateral-medial small amplitude (movement distance) and amplitude limit available respecting the patient's pain.

Reassessments occurred at the end of fifteen sessions following the same initial evaluation parameters.

\section{ANALYSIS OF RESULTS}

The data are presented in tables describing the variables sex, age, occupation, affected limb, impairment of time, comorbidities and medication use.

The pain score variables, degree of ROM and score of functional capacity measured in the evaluation and reevaluation are compared descriptively for each volunteer and presented as changes in range in the evaluation and reevaluation (DAV - Rv) and Percentage change ( $\triangle \%$ ) to each variable given by equation 1 :

$$
\Delta \%=\frac{\mathrm{Rv}-\mathrm{Av}}{\mathrm{Av}} \cdot 100
$$

(Equation 1)

$A v=$ measured value of the variable in the assessment;

$\mathrm{RV}=$ measured value of the variable in the revaluation.

Specifically, to assess the level of pain, the data are presented in three stages: an initial assessment at the end of the seventh session and at the end of 15 sessions.

\section{RESULTS}

The volunteers in the study had a mean age of 59.25 years, three female. It was observed in three volunteers the affected limb was dominant, and that all were in the first phase of disease with an average of 6 months $^{(6)}$. Data on medication use, comorbidities and profession are shown in Table 1.

As for the etiology, volunteers 1 and 3 reported having suffered mechanical trauma to the shoulder area with consequent immobilization period. Voluntary 2 chronicled the emergence of subdeltoid bursitis that has not had physiotherapy sessions, progressing to the immobility of the joint as a form of protection, the same voluntary exposed be diabetic type I. In contrast, voluntary 4 reported that symptoms began insidiously with no no prior trauma to justify the appearance of CA.

In response to therapy by the Maitland method, the four volunteers had decreased pain symptoms. The scores at baseline, measured by VAS, ranged between 9-10 points, occurring at the end of treatment improved from 55.5 to $80 \%$ of the pain level (Figure 1).
The Figure 2 shows the goniometric data collected from participants in the evaluation and re-evaluation and their percentage of improvement. After the intervention was increased ROM, in particular for bending with gains of more than $100 \%$. But the internal rotation in volunteers 1 and 3 had won lower than the others.

The total scores of SPADI questionnaire in the evaluation ranged from 80.00 to 94.61 with the pain domain showing the highest scores ( 90.00 to 94.00 ). At the end of the interventions was observed evolution of functional capacity through the improvement percentage between 27.9 to $91.9 \%$ (Figure 3).

Table 1. Holders users Adhesive capsulitis characterization of shoulder submitted to Maitland method.

\begin{tabular}{|c|c|c|}
\hline Variables & $\begin{array}{l}\text { Total } \\
n=4\end{array}$ & $\%$ \\
\hline \multicolumn{3}{|l|}{ Age (years) } \\
\hline$\geq 50 \leq 60$ & 4 & 100 \\
\hline \multicolumn{3}{|l|}{ Genre } \\
\hline Male & 1 & 25 \\
\hline Female & 3 & 75 \\
\hline \multicolumn{3}{|l|}{ Affected side } \\
\hline Dominant & 3 & 75 \\
\hline Non-dominant & 1 & 25 \\
\hline \multicolumn{3}{|l|}{ Condition time (months) } \\
\hline$\geq 1 \leq 6$ & 3 & 75 \\
\hline$\geq 7 \leq 12$ & 1 & 25 \\
\hline \multicolumn{3}{|l|}{ Use of pain medications } \\
\hline Antiinflamatories & 2 & 50 \\
\hline \multicolumn{3}{|l|}{ Comorbidities } \\
\hline Diabetes Mellitus & 1 & 25 \\
\hline \multicolumn{3}{|l|}{ Profession } \\
\hline Home & 1 & 25 \\
\hline Retired & 2 & 50 \\
\hline (driver/clerk of store)Public Agent & 1 & 25 \\
\hline
\end{tabular}

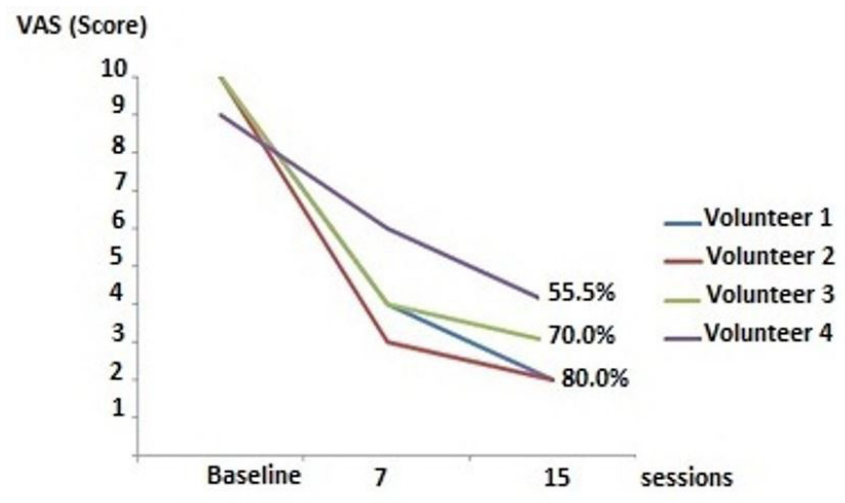

Figure 1. Pain scores in the evaluation measures and after the 7th and 15th sessions by Visual Analogue Scale (VAS) and their percentage change of the volunteers. 
Flexion

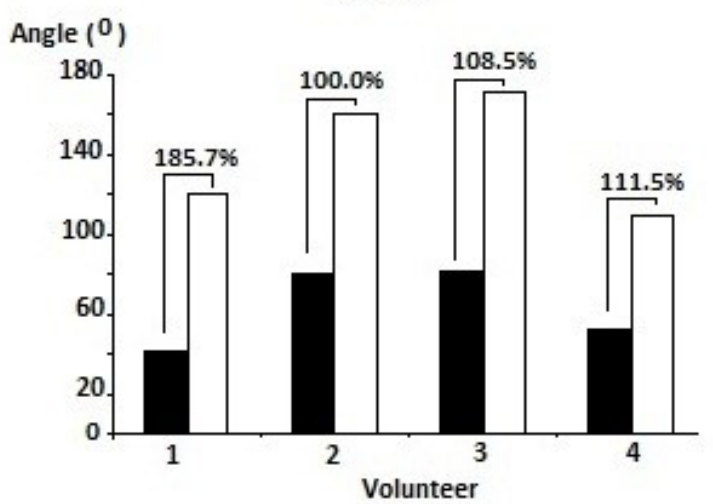

External rotation

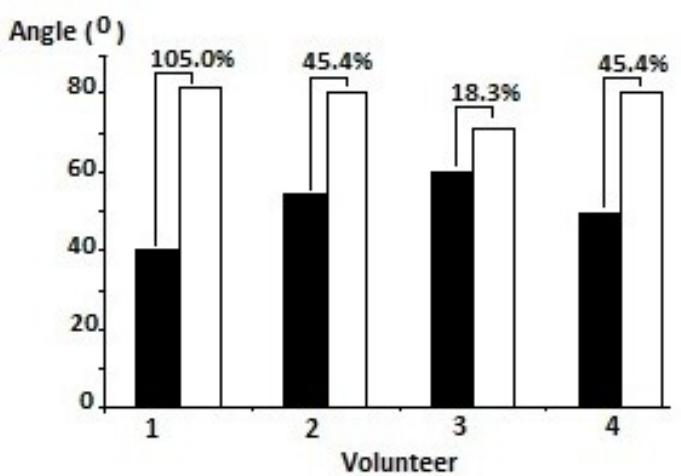

Evaluation
Abduction

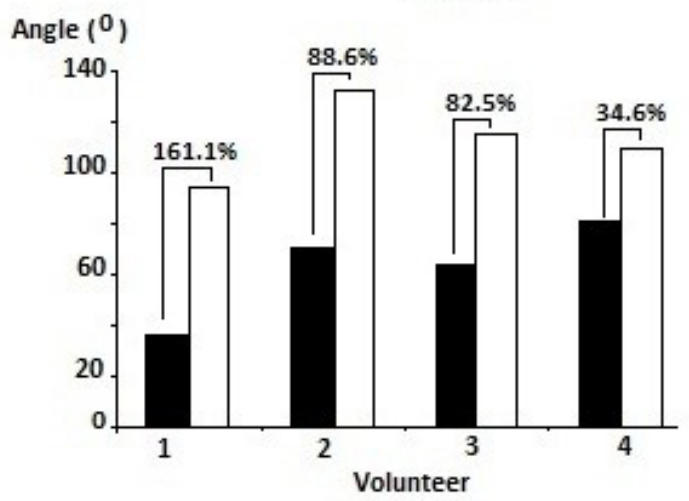

Internal rotation

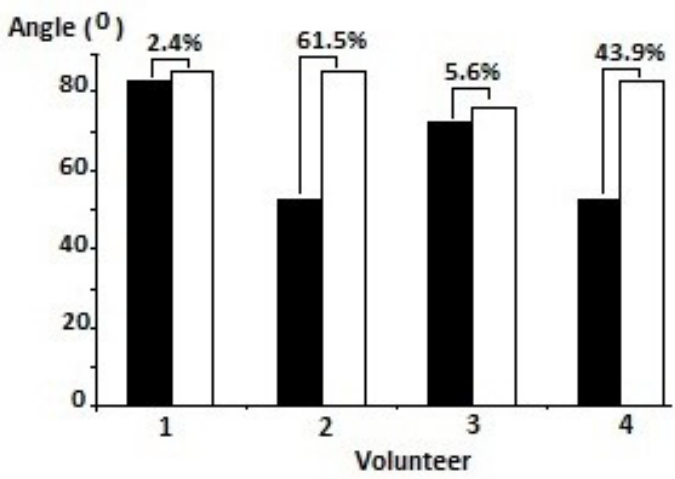

Revaluation

Figure 2. Goniometric measures for flexion, abduction, external and internal rotation of the affected limb and percentage change of the measured angles between the evaluation and reevaluation of the volunteers.

\section{DISCUSSION}

Ortiz -Lucas et al. ${ }^{(6)}$ in a systematic review, found that the total of 246 patients were 138 women, 78 men and 30 did not have the sex specified. However, despite the preponderance of female patients, the influence of gender has not yet been elucidated. ${ }^{(19)}$

Regarding the etiology, primary AC, there is loss of assets and liabilities movements of the glenohumeral joint insidiously progressive ${ }^{(20)}$; since the secondary etiology has a variety of clinical conditions that may contribute to its onset, including prolonged immobilization of the shoulder for reasons such as cuff injury, bursitis, and traumas. ${ }^{(21)}$

Studies ${ }^{(22,23)}$ indicate increased prevalence of CA in diabetics may reach $30 \%$ in insulin-dependent patients. Bridgman (1972) ${ }^{(24)}$ was the first to perform this association, however in relation to the voluntary 3 is not possible to state that diabetes is involved, due to the history trauma.

The involvement of the dominant hand is present between $40-42 \%$ of cases and bilaterally $7 \%{ }^{(25.26)}$, since the involvement of the non-dominant occurs in $20-30 \% .{ }^{(27)}$ In this context two volunteers reported pain at the onset of contralateral shoulder, but the pain could result from the overload member and none sought medical care for diagnosis during the interventions.

The joint mobilization in the movement boundary associated with scapular mobilization was effective when evaluated ROM and shoulder functional capacity. ${ }^{(16)}$ Comparison of joint mobilization of low grade (I and II) and high grade (III and IV) was shown by randomized clinical trial that the high-grade group was superior in all variables (pain, ROM, functional capacity and quality of life) with significant results ${ }^{(28)}$ because the oscillations of great amplitude stimulate more mechanoreceptors being more effective in reducing pain. ${ }^{(29)}$

The effect of anterior and posterior handling on the range of external rotation in patients with CA were analyzed using a randomized clinical trial. The volunteers received the same intervention protocol consisted of applying therapeutic ultrasound joint mobilization and exercise for the upper limb for 6 sessions, 2 to 3 times per week. The treatment differed 

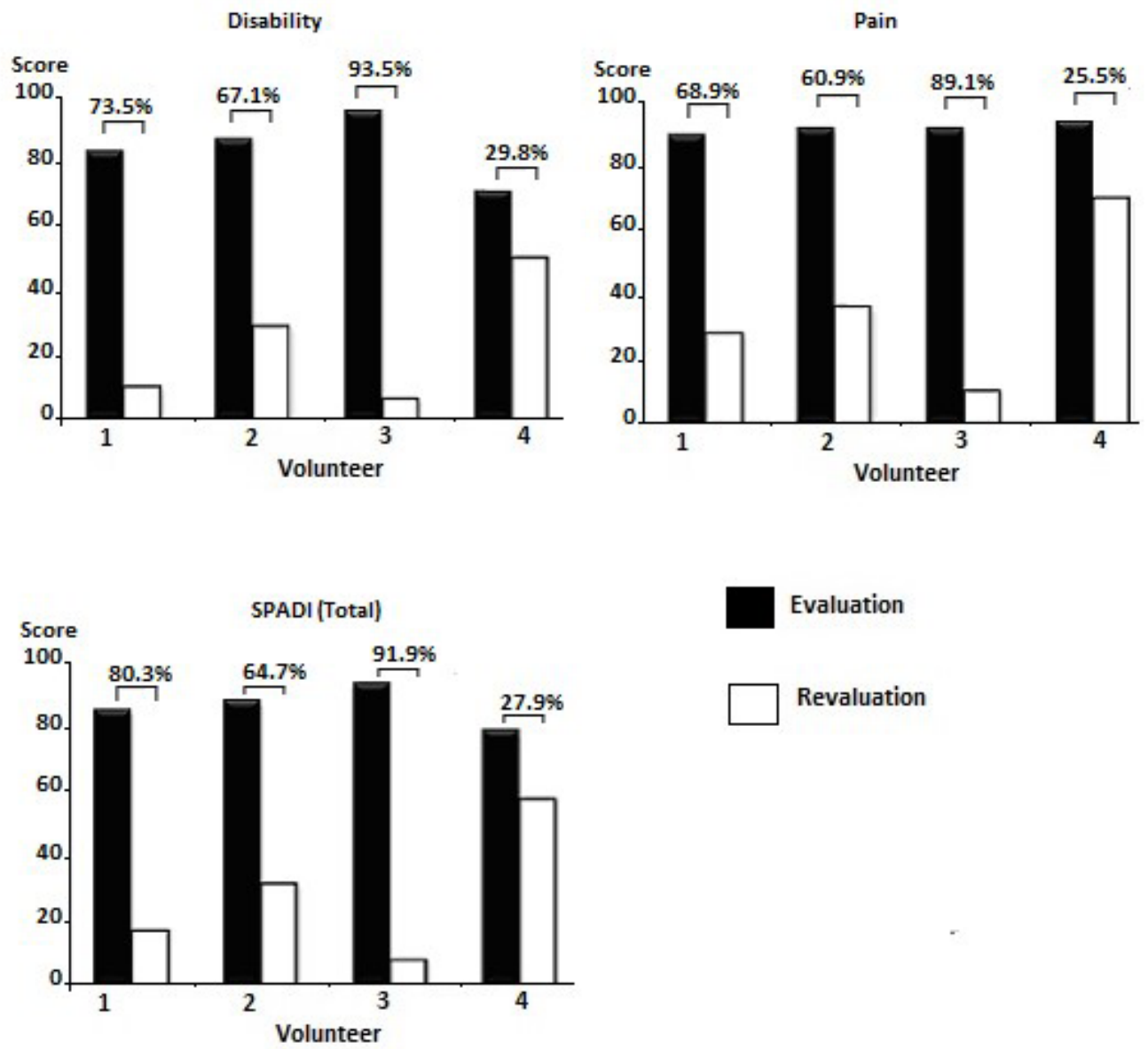

Evaluation

Revaluation

Figure 3. Ratings Pain Index and Shoulder Disability (SPADI) during evaluation and reevaluation with percentage variation of their volunteers.

in the sense of manipulation in the previous in previous (MA group) and posterior (MP group). The external rotation was measured by goniometer and pain was punctuated by VAS and the results showed that the MP group had significantly improved ROM unlike the AM group. Both groups showed significant results regarding pain and there were no significant differences when comparing the two. ${ }^{(30)}$

This study differed methodologically from our study because the joint mobilization was the only intervention used in all movements and plans which may have contributed to the gains in the other motion arcs.

The volunteers 1 and 3 had percentage of improvement to lower internal rotation to the other during anamnesis noted that the etiology of both was traumatic with rotator cuff components injury that was not followed under medical supervision for later repair that may have affected the evolution of the same.

Among the participants voluntary 4 obtained the lowest percentage of improvement in the variables collected, this was probably the difficulty presented in the realization of the protocol. Due to the overweight was unable to switch to DD and DV series are all applied with the same sitting undermining the provision of assistance.
The results were positive in this study using only the Maitland method, probably due to the higher number of sessions, the short break between each session and the AC phase in which volunteers were. Note that the sample size, the use of anti-inflammatories for two volunteers are limiting factors in the study. The scarcity of studies evaluating the effectiveness of Maitland method alone complicated the discussion of this work, so this is one of the contributions of this study, decreasing the gap in the literature on the clinical evidence method.

\section{CONCLUSION}

The joint manipulation by the Maitland method obtained beneficial effects in the treatment of shoulder CA with decreasing pain, increasing range of motion and functional capacity. However, there is need for further research with larger sample to determine efficacy of the method.

\section{AUTHOR'S CONTRIBUTIONS:}

MRTO LSGN VCSB: Collect the data, conceived and designed the experiments MFAB AGCC MRG: Review the literature, wrote and corrected the article SLC MBGJ: Analysed the data

MGRA: Guided the work, conceived and designed the experiments. 


\section{CONFLICTS OF INTEREST:}

There are no conflicts of interest.

\section{AUTHOR DETAILS}

${ }^{1}$ Instituto de Medicina Integral Professor Fernando Figueira - IMIP, Recife (PE), Brazil

2 UFPE- Universidade Federal de Pernambuco, Recife (PE), Brazil.

${ }^{3}$ Department of Physiotherapy, Universidade Federal da Paraíba, João Pessoa (PB), Brazil.

${ }^{4}$ Department of Physiotherapy, Coordinator of the Motor Control Laboratory and Postural Balance, Universidade Federal de Sergipe- UFS, Aracajú (SE), Brazil.

\section{REFERENCES}

1. Facci, LM. Síndromes Dolorosas do Ombro: Análise de sua Incidência e Características. Arq. Ciênc. Saúde UNIPAR. 2000;4(3):195-200.

2. Tasto JP, Elias DW. Adhesive capsulitis. Sports Med Arthrosc Rev. 2007;15:216-21.

3. Ferreira Filho, AA. Capsulite Adesiva. Rev Bras Ortop. 2005;40(10):565-74.

4. Kisner C, Colby LA. Exercícios terapêuticos: fundamentos e técnica. 5 th ed. Barueri(SP): Manole; 2005.

5. Ryans I, Montgomery A, Galway R, Kernohan WG, McKane R. A randomized controlled trial of intra-articular triamcinolone and/or physiotherapy in shoulder capsulitis. Rheumatology 2005;44:529-535.

6. Ortiz-Lucas M, Hijazo-Larrosa S, Estébanez-De-Miguel E. Capsulitis adhesiva del hombro: una revisión sistemática. Fisiot 2010;32(5):229-235.

7. Kelley MJ, Shaffer MA, Kuhn JE, Michener LA, Seitz AL, UhI TL, et al. Shoulder Pain and Mobility Deficits: Adhesive Capsulitis. J Orthop Sports Phys Ther 2013;43(5):A1-A31.

8. Araujo AGS, Meurer TL. Protocolos de tratamento da capsulite adesiva metanálise. Cinergis. 2012;13(3):9-15.

9. Herrero MTV, García LC, González AAL, Torre MVLI. El hombro y sus patologías en medicina del trabajo. Semergen. 2009;35(4):197-202.

10. Ciccone $C C$, Oliveira MAD, Hildebrand AS. Revisão bibliográfica da anatomia de ombro e da Capsulite adesiva para futura abordagem na terapia manual de Maitland. Rev bras fisioter. 2007;10(2):285-290.

11. Barboza RI, Goes R, Mazzer N, Fonseca MCR. A influência da Mobilização Articular nas tendinopatias dos músculos bíceps braquial e supraespinhal. Rev Bras Fisioter. 2008;12(4):298-303.

12. Resende MA, Venturini C, Penido MM, Bicalho LI, Peixoto GHC, Chagas MH. Estudo da confiabilidade da força aplicada durante a mobilização articular antero-posterior do tornozelo. Rev. bras. Fisioter. 2006;10(2):199-204.

13. Corrigan B, Maitland GD. Transtornos musculoesqueléticos da coluna vertebral. Rio de Janeiro (RJ): Revinter; 2005.

14. León NR, Pilat A. El Concepto de Maitland. Terap Man Venez. 2001;5(8).
15. Yang JI, Jan $\mathrm{MH}$, Chang CW, Lin JJ. Effectiveness of the end-range mobilization and scapular mobilization approach in a subgroup of subjects with frozen shoulder syndrome: A randomized control trial. Manual Therap. 2012;17:47-52.

16. Vogt L, Segieth C, Banzer W, Himmelreich H. Movement behaviour in patients with chronic neck pain. Physiother Res Int. 2007;12(4):206-12.

17. Martins J, Napoles BV, Hoffman CB, Oliveira AS. Versão brasileira do Shoulder Pain and Disability Index: tradução, adaptação cultural e Confiabilidade. Rev Bras Fisioter. 2010;14(6):527-36.

18. Maitland GD, Banks K, English K, Hengeveld E. Manipulação vertebral de Maitland. 6th ed. Rio de Janeiro (RJ): Medsi; 2003.

19. Sheridan MA, Hannafin JA. Upper Extremity: Emphasis on Frozen Shoulder. Orthop Clin. 2006;37:531-539.

20. Cleland J, Durral CJ. Physical Therapy for Adhesive Capsulitis: Systematic review. Physiother. 2002;88(8):450-457.

21. Tasto JP, Elias DW. Adhesive capsulitis. Sports Med Arthrosc Rev. 2007;15:216-21.

22. Thomas SA, McDougall C, Brown IDM, Jaberoo MC, Stearns A, Ashraf $R$, et al. Prevalence of symptoms and signs of shoulder problems in people with diabetes mellitus. J Shoulder Elbow. 2007;16(6):748-751.

23. Lorbach O, Anagnostakos K, Scherf C, Seil R, Kohn D, Pape D. Nonoperative management of adhesive capsulitis of the shoulder: Oral cortisone application versus intra-articular cortisone injections. J Shoulder Elbow Surg. 2010;19:172-179.

24. Bridgman JF. Periarthritis of the shoulder and diabetes mellitus. Ann Rheum Dis. 1972;31:69-71.

25. Levine WN, Kashyap CP, Bak SF, Ahmad CS, Blaine TA, Bigliani LU. Nonoperative management of idiopathic adhesive capsulitis. J Shoulder Elbow Surg. 2007;16(5):569-573.

26. Vermeulen HM, Rozing PM, Obermann WR, Cessie S, Vlieland V. Comparison of High-Grade and Low- Grade Mobilization Techniques in the Management of Adhesive Capsulitis of the Shoulder: Randomized Controlled Trial. Phys Ther. 2006;86:355-368.

27. Griggs SM, Ahn A, Green A. Idiopathic adhesive capsulitis: A prospective functional outcome study of nonoperative treatment. J Bone Joint Surg. 2000;82(10):1398.

28. Vermeulen HM, Rozing PM, Obermann WR, Cessie S, Vlieland V. Comparison of High-Grade and Low- Grade Mobilization Techniques in the Management of Adhesive Capsulitis of the Shoulder: Randomized Controlled Trial. Phys Ther. 2006;86:355-368.

29. Krouwel O, Hebron C, Willett E. An investigation into the potential hypoalgesic effects of different amplitudes of PA mobilisations on the lumbar spine as measured by pressure pain thresholds (PPT). Man Therap. 2010;15:7-12.

30. Johnson AJ, Godges JJ, Zimmerman GJ, Ounanian LL. The Effect of Anterior Versus Posterior Glide Joint Mobilization on External Rotation Range of Motion in Patients With Shoulder Adhesive Capsulitis. J Orthopaedic \& Sports Phys Ther. 2007;23(3):88-99. 